\title{
Estrategias para la gestión integrada y sostenible del recurso hídrico en el municipio de Pauna (Boyacá)
}

Strategies for the Integrated and Sustainable Management of Water Resources in the Municipality of Pauna (Boyacá)

Estratégias para a gestão integrada e sustentável do recurso hídrico no município de Pauna (Boyacá)

\section{Juan Villalobos-Gualteros ${ }^{1}$ Lida Ruiz-Martínez ${ }^{2}$}

Recibido: julio 5 del 2018

Aprobado: octubre 23 del 2018 Disponible en línea: enero 15 del 2019

Cómo citar este artículo:

J. Villalobos-Gualteros y L. Ruiz-Martínez, "Estrategias para la gestión integrada y sostenible del recurso hídrico en el municipio de Pauna (Boyacá)", Revista Ingeniería Solidaria, vol. 25, n. ${ }^{\circ} 1,2019$. DOI: https://doi.org/10.16925/2357-6014.2019.01.03

Artículo de investigación. https://doi.org/10.16925/2357-6014.2019.01.03

1 Universidad Libre de Colombia, Bogotá, Colombia

ORCID: https://orcid.org/0000-0001-8930-7831

Correo electrónico: Juand.villalobosg@unilibrebog.edu.co

2 Universidad Libre de Colombia, Bogotá, Colombia

ORCID: https://orcid.org/0000-0002-0236-425x 


\section{Resumen}

Introducción: el presente artículo es producto de la investigación "Estrategias para gestión integrada y sostenible del recurso hídrico", realizada durante 2017 y 2018 en el municipio de Pauna, ubicado en el occidente del departamento de Boyacá (Colombia).

Problema: el uso insostenible del recurso hídrico por parte de la población del municipio constituye una problemática de prioridad social y ambiental; el agua en el municipio se ha utilizado con fines cada vez más numerosos, en aras de atender las necesidades que surgen del desarrollo económico y turístico.

Objetivo: formular estrategias de gestión para el uso eficiente del agua en los sectores de consumo residencial, comercial y turístico del municipio.

Metodología: el uso eficiente del agua implicó comprender y caracterizar los hábitos, además de los sectores de consumo, y verificar la calidad del agua en aras de crear estrategias de gestión ambiental mediante encuestas descriptivas de tipo abierto. Para revisar la variación de la calidad del agua se tomaron muestras puntales. Resultados: el uso insostenible del recurso hídrico está ligado al uso de grifería obsoleta, a la creciente demanda del agua debido al desarrollo y al turismo, y al mantenimiento general de balnearios.

Conclusión: con este proyecto se generó un cambio en el comportamiento del uso hacia la eficiencia y la modificación de prácticas de los usuarios, a fin de favorecer la sustentabilidad de los habitantes.

Originalidad: se formularon estrategias de gestión integrada y sostenible del recurso hídrico por primera vez en el municipio de Pauna.

Limitaciones: la carencia de información suministrada por el municipio y el acceso a los puntos de muestreo.

Palabras clave: contaminación, desarrollo, necesidades, Pauna, recurso hídrico, sustentabilidad.

\section{Abstract}

Introduction: This article is the outcome of a research called "Strategies for integrated and sustainable management of water resources", carried out during 2017 and 2018 in the municipality of Pauna, located in the west of the Department of Boyacá.

Problem: The unsustainable use of water resources by the population of the municipality makes it a problem of social and environmental priority. The water in the municipality has been used for more and more numerous purposes that seek to meet the needs that arise from economic and tourist development.

Objective: To formulate management strategies for the efficient use of water in the residential, commercial and tourist consumption sectors of the municipality.

Methodology: The efficient use of water implies understanding and characterizing the habits, in addition to the consumption sectors, and verifying the water quality, to create environmental management strategies through open type descriptive surveys, and sampling struts were used to assess the variation in water quality.

Results: The unsustainable use of water resources is linked to the use of obsolete faucets, increasing demand for development and tourism, and general maintenance of spas.

Conclusion: This project seeks to generate a change in the behavior of the use towards efficiency, and the modification of the practices of the users to favor the sustainability of the inhabitants.

Originality: Strategies of integrated and sustainable management of water resources are formulated for the first time in the municipality of Pauna.

Limitations: The lack of information provided by the municipality, and access to the sampling points.

Keywords: pollution, development, needs, Pauna, water resource, sustainability. 


\section{Resumo}

Introdução: o presente artigo é produto da pesquisa “Estratégias para gestão integrada e sustentável do recurso hídrico", realizada durante 2017 e 2018 no município de Pauna, localizado no ocidente do Departamento de Boyacá (Colômbia).

Problema: o uso insustentável do recurso hídrico por parte da população do munícipio constitui uma problemática de prioridade social e ambiental; a água no município é utilizada com fins cada vez mais numerosos, para efeitos de atender as necessidades que surgem do desenvolvimento econômico e turístico.

Objetivo: formular estratégias de gestão para o uso eficiente da água nos setores de consumo residencial, comercial e turístico do munícipio.

Metodologia: o uso eficiente da água implicou compreender e caracterizar os hábitos, além dos setores de consumo, e verificar a qualidade da água com o objetivo de criar estratégias de gestão ambiental por meio de questionários descritivos de tipo aberto. Amostras pontuais foram recolhidas para revisar a variação da qualidade da água.

Resultados: o uso insustentável do recurso hídrico está ligado ao uso de tubulação obsoleta, à crescente demanda de água devido ao desenvolvimento e ao turismo e à manutenção geral de balneários.

Conclusão: com este projeto, gerou-se uma mudança no comportamento do uso com respeito à eficiência e à modificação de práticas dos usuários, a fim de favorecer a sustentabilidade dos habitantes.

Originalidade: estratégias de gestão integrada e sustentável do recurso hídrico foram formuladas pela primeira vez no município de Pauna.

Limitações: a carência de informação fornecida pelo município e o acesso aos pontos de amostragem.

Palavras-chave: contaminação, desenvolvimento, necessidades, Pauna, recurso hídrico, sustentabilidade.

\section{Introducción}

La gestión integrada del recurso hídrico (GIRH) es un concepto relevante que se encuentra basado en los usos del agua en tanto situaciones demandantes del recurso. Ejemplos de ellas pueden ser las actividades turísticas y de desarrollo; el funcionamiento de empresas con ánimo de lucro; y en general, todas aquellas que involucran al ser humano, las cuales conllevan una inevitable falta de agua de buena calidad, en condiciones óptimas para el uso de la población.

El crecimiento de la población, así como el aumento de la actividad económica y los estándares de vida, han sido causantes de la competencia por obtener el recurso hídrico en óptimas condiciones y con estándares de alta calidad. Las preocupaciones actuales con respecto a la variabilidad y el cambio climático requieren una gestión del recurso hídrico que tenga un enfoque participativo y una visión de producción más limpia, con lo cual se prevengan inundaciones y sequías más intensas [1].

Los beneficios de la GIRH son importantes para una región en términos ambientales puesto que, al determinar la importancia de las necesidades medioambientales en la distribución del agua, es posible beneficiarse de la aplicación de una formulación 
integrada y sostenible para el recurso hídrico de los ecosistemas. En la actualidad, es normal que estas necesidades no estén representadas y dispuestas en la mesa de negociación y toma de decisiones de las administraciones. En este sentido, la GIRH puede tener relevancia frente al sector, al generar buenas prácticas y cambiar hábitos entre otros usuarios de las necesidades de los ecosistemas y de los beneficios que estos les genera [2].

El control y la conservación de un ecosistema brindan, en términos teóricos, un marco para la GIRH que enfoca más su atención en una formulación integrada de estrategias para la gestión del recurso hídrico. Esto comprende temáticas importantes, tales como reforestación, buen manejo de la tierra, control y prevención de la erosión del suelo, control de contaminación y flujos ambientales; de igual manera, se provee una alternativa para la perspectiva de competencia de sectores más pequeños, que puede reunir a los interesados a fin de desarrollar un punto de vista compartido.

El sector agrícola es el mayor usuario de agua, así como el generador principal de la contaminación del recurso superficial y subterráneo de una cuenca hidrográfica. En este sentido, la reducción en la repartición de agua para fines agrícolas trae consecuencias de largo alcance en los ámbitos social y económico. A través de la GIRH se quiere impulsar a quienes realizan la gestión, o la formulan, a tener una visión más allá de la economía y desarrollo del sector, además de tener en cuenta las implicaciones de las decisiones sobre el uso eficiente del recurso hídrico, el medio ambiente y la igualdad social [1].

Al poner en consideración y en participación colectiva a todos los sectores en el proceso de toma de decisiones, la GIRH plantea reflejar el valor relacionado del agua para la sociedad y el bienestar como un todo; esto puede influir de forma positiva en la producción de alimentos y en la salud, a la vez que puede contribuir a la reducción de la pobreza y la igualdad de género. Así entonces, el objetivo principal de la GIRH es ofrecer un planteamiento totalmente integrado que busca aumentar la productividad de forma sostenible del agua.

El municipio de Pauna (departamento de Boyacá, Colombia) ostenta una alta actividad en lo que atañe al turismo por sus atracciones naturales y ecológicas, a lo que se suma su amplia infraestructura en relación con este sector (representada por emplazamientos como hoteles y balnearios). Las estrategias de gestión integrada y sostenible del recurso hídrico se encuentran en función de las prácticas de ingeniería y desarrollo del municipio de Pauna. Este es un municipio con una visión de progreso social, cultural, territorial, de convivencia y productividad, y enfocado en un desarrollo sostenible, pero el nivel de conciencia de sus habitantes y dirigentes sobre el uso eficiente del agua es bajo [3]. 


\subsection{Antecedentes - estado del arte en el ámbito nacional}

- Según un estudio realizado en la Universidad Militar Nueva Granada (2011), el uso eficiente del agua incluye toda actividad relacionada con el uso racional del recurso; en dicha investigación se plantea la necesidad de formular medidas que permitan reducir el uso de agua en cualquier proceso o actividad que permita su conservación y mejoramiento. El uso eficiente, uno de los principios contemplados para el manejo integrado del recurso hídrico, se efectúa mediante programas desarrollados por etapas: recopilación de la información, diagnóstico, caracterización, análisis de resultados, formulación y diseño. Estas actividades se encuentran enfocadas a optimizar el recurso hídrico y hacer uso sostenible del mismo, mediante la identificación y caracterización de aquellos eventos que por su naturaleza producen efectos negativos sobre el medio, de tal manera que se establezcan medidas de control y mitigación adecuadas (tabla 1) [4].

Tabla 1. Estrategias de uso eficiente de agua

\begin{tabular}{|c|c|c|c|c|}
\hline Programa & $\begin{array}{l}\text { Proyectos o } \\
\text { estrategias }\end{array}$ & Objetivos & Metas & Indicadores \\
\hline \multirow{3}{*}{$\begin{array}{l}\text { Gestión de segui- } \\
\text { miento }\end{array}$} & $\begin{array}{l}\text { Gestionar la obten- } \\
\text { ción de la concesión } \\
\text { de agua. }\end{array}$ & $\begin{array}{l}\text { Adquisición de la } \\
\text { concesión de agua } \\
\text { del río por parte } \\
\text { de la autoridad } \\
\text { ambiental. }\end{array}$ & $\begin{array}{l}\text { Obtener la conce- } \\
\text { sión de agua, emiti- } \\
\text { da por corporación } \\
\text { pertinente. }\end{array}$ & $\begin{array}{l}\text { Número de conce- } \\
\text { siones de agua / nú- } \\
\text { mero de solicitudes } \\
\text { de concesión. }\end{array}$ \\
\hline & $\begin{array}{l}\text { Revisión y manteni- } \\
\text { miento del sistema } \\
\text { de micromedición. }\end{array}$ & $\begin{array}{l}\text { Revisión y manteni- } \\
\text { miento del sistema } \\
\text { de distribución y } \\
\text { micromedición } \\
\text { existente en la red } \\
\text { de distribución. }\end{array}$ & $\begin{array}{l}\text { Disminuir en un } \\
10 \% \text { el consumo de } \\
\text { agua, de acuerdo } \\
\text { con el IANC por año, } \\
\text { en } 6 \text { meses. }\end{array}$ & $\begin{array}{l}\text { Volumen agua } \\
\text { captada / volumen } \\
\text { agua distribuida. }\end{array}$ \\
\hline & $\begin{array}{l}\text { Control y reducción } \\
\text { de pérdidas. }\end{array}$ & $\begin{array}{l}\text { Disminuir las pér- } \\
\text { didas del sistema } \\
\text { de acueducto por } \\
\text { medio del control } \\
\text { de fugas en la } \\
\text { conducción y redes } \\
\text { de distribución. }\end{array}$ & $\begin{array}{l}\text { Reducir las fugas } \\
\text { en el sistema de } \\
\text { acueducto en un } \\
30 \% \text { en } 1 \text { año. }\end{array}$ & $\begin{array}{l}\text { Porcentaje de re- } \\
\text { ducción de pérdidas } \\
\text { por año. }\end{array}$ \\
\hline
\end{tabular}

Fuente: elaboración propia 
6 Estrategias para la gestión integrada y sostenible del recurso hídrico en el municipio de Pauna (Boyacá)

Con una investigación adelantada en el municipio de El Colegio (departamento de Cundinamarca) se pretendió elaborar un programa de ahorro y uso eficiente del agua para la empresa Empucol; con este se buscaba dar solución a una problemática que venía de años atrás, puesto que el recurso hídrico estaba siendo afectado por las diferentes actividades antrópicas. Para elaborar el programa, se identificaron la situación y el consumo actual del recurso en el municipio, al tiempo que se revisaron las estrategias utilizadas en otros programas; asimismo, se revisaron los documentos suministrados por la Alcaldía Municipal y la Empresa de Servicios Públicos (ESP), "con las tarifas de los servicios básicos, el plan maestro de acueducto y alcantarillado, el plan de saneamiento y manejo de vertimientos, entre otros, para formular así las posibilidades de ahorro" [5].

El municipio de Arcabuco (Boyacá), a su turno, y en conjunto con la Pontificia Universidad Javeriana, desarrolló un proyecto de investigación para establecer estrategias de gestión ambiental, a partir de la formulación de un esquema de pago de servicios ambientales (PSA) para regular el recurso hídrico en la Quebrada La Colorada del municipio: "el proyecto de investigación es una aproximación conceptual y metodológica que permitió proponer una estrategia de gestión ambiental a través de la formulación de un esquema de pago por servicios ambientales (PSA) para la regulación del recurso hídrico en términos de calidad y cantidad; para esto se hizo necesaria la revisión de información secundaria y la aplicación de una encuesta que permitió establecer que el esquema de PSA se enmarca en un ecosistema de bosque andino en el cual se identificaron elementos biofísicos y socioeconómicos relevantes" [6].

Sumado a lo anterior, en 2012 se realizó en la Universidad Tecnológica de Pereira, una investigación sobre los hábitos de consumo del recurso hídrico en las instalaciones de la Facultad de Ciencias Ambientales, a través de la cual se buscó formular estrategias de uso eficiente y ahorro del agua, en respuesta al alto consumo de la comunidad académica de esa institución. La investigación representó un aporte a la gestión del agua para centros educativos, lo cual implicó un control eficiente y eficaz en los sistemas de distribución y por parte de los consumidores [7]. En palabras de su autor, "a fin de iniciar un proceso de gestión del recurso hídrico en la Universidad Tecnológica de Pereira y, tomando como caso de estudio la Facultad de Ciencias Ambientales, se hace necesario identificar los usuarios del agua y sus hábitos de consumo, con el fin de generar procesos de comprensión y herramientas de gestión en torno a la uso del agua, que conduzcan a la reducción o prevención de fugas en la red de distribución al igual que un cambio en la actitud de la población frente al consumo responsable" [7]. 
Por otra parte, según la investigación realizada por el programa de Ingeniería de Recursos Naturales y del Medio Ambiente de la Universidad del Valle (2007), la variabilidad y el cambio climáticos se han asociado a cambios globales o en el régimen climático; y han mostrado notables efectos en el ciclo hidrológico. La escasez del agua, los cambios en la intensidad de la precipitación y la variabilidad de eventos externos están cobrando una importancia significativa. Como consecuencia del aumento de la temperatura del aire y cambios en la precipitación, el cambio climático está incrementando la competencia por el agua dulce y está afectando los procesos y regímenes hidrológicos.

"La necesidad de adaptarse al cambio climático aparece cuando se están produciendo grandes cambios económicos y sociales, que influyen en la utilización de los recursos hídricos. El cambio climático está aumentando la vulnerabilidad en sistemas hídricos mal administrados, haciendo urgente la necesidad de construir una nueva visión de gestión de los recursos hídricos" [8].

La investigación da lugar al concepto y metodología de la gestión integrada del recurso hídrico como estrategia de adaptación al cambio climático, en tanto con ella se determina que "la gestión integrada del recurso hídrico es un proceso de desarrollo coordinado de los recursos naturales, que maximiza el bienestar de la comunidad en general de manera equitativa y sin comprometer la sostenibilidad de los ecosistemas vitales. Esto supone superar los viejos paradigmas donde los enfoques referentes al suministro de agua tienen mayor difusión que aquellos referidos a la demanda" [8].

"(...) Kundzewicz \& Mata (2004) recogen algunas de las estrategias de adaptación propuestas mundialmente:

- Incremento de la habilidad de intercambio entre los sectores.

- Usos de mecanismos de mercadeo para aumentar la eficacia del uso del agua.

- Incorporación de cambios potenciales en la demanda y oferta, en la planificación y en el diseño de infraestructura.

- Reubicación de comunidades y estructura fuera de las planicies de inundables.

- Identificación de formas sostenibles de manejar la oferta disponible, incluyendo agua subterránea, superficial y los efluentes.

- Restauración y mantenimiento de las fuentes de agua para el manejo de calidad y la cantidad del agua" [8]. 
Según Tarquino, "los avances en el conocimiento han llevado las propuestas para la gestión de los recursos hídricos, como respuesta a la denominada "crisis del agua", la cual es una de las mayores preocupaciones del planeta. La adecuada gestión de estos recursos forma además parte de las propuestas para alcanzar los Objetivos de Desarrollo del Milenio (ODM) de las Naciones Unidas, especialmente el objetivo de reducir la pobreza y el hambre que sufren buena parte de la población mundial. Así, el ser humano y el ambiente se convierten en el centro de las propuestas de gestión del agua, no solo por su efecto sobre la salud sino por su influencia sobre el bienestar y el desarrollo de los pueblos. La ingeniería del agua debería entonces dar un vuelco de tal forma que la tecnología sea una respuesta a las necesidades sociales, ambientales y económicas de la sociedad, bajo criterios de eficiencia y sostenibilidad" [9].

Un estudio adelantado desde la Universidad de la Salle en 2007, enfocado al ahorro y uso eficiente del recurso hídrico en una empresa del sector de aceites y grasas vegetales en la ciudad de Bogotá, estableció la implementación de planes de ahorro y uso eficiente del agua.

"La empresa objeto de estudio, se encuentra dentro del grupo de industriales del sector oleaginoso, que implementan planes de ahorro y uso eficiente del agua. Estos planes de ahorro buscan reducir los consumos y usos excesivos de agua dentro de las diferentes áreas dentro del proceso de refinación de grasas y aceites, ejecutando estrategias de mejora y capacitando al personal en el uso racional de este recurso" [10].

Como primera medida, se realizó control y seguimiento a los 16 contadores de agua ubicados en distintas áreas de la planta, en aras de determinar las zonas o puntos críticos del consumo del recurso. De modo simultáneo se realizaron capacitaciones para concientizar al personal de la empresa en el uso eficiente de este, a través de las cuales se involucró a estas personas en el desarrollo de las estrategias implementadas como parte del proceso. Además, se efectuó un levantamiento de planos para conocer la distribución del sistema y encontrar las zonas donde no se realiza la recuperación de condensados.

"Al proponer estrategias de ahorro se tuvo en cuenta la producción de vapor, el uso del agua de calderas, la recirculación de condensados, estado de las tuberías de transporte de agua y vapor (recubrimientos térmicos), lavados, instalación de dispositivos ahorradores y por consiguiente la disminución en la generación y tratamiento de las aguas residuales, optimizando el funcionamiento de la planta de tratamiento y disminuyendo los costos de operación" [10]. 


\subsection{Antecedentes - estado del arte en el ámbito internacional}

La sequía en el suroeste de Victoria (uno de los seis estados que conforman la Mancomunidad de Australia) [11] y en todo el mundo ha causado la necesidad de mitigar el consumo de agua para garantizar el suministro de dicho recurso en el futuro. Con esto, la Facultad de Ciencias de Vida y del Medio Ambiente, junto a la Facultad de Ciencia y Tecnología de la Deakin University, formularon estrategias eficientes de cambio en el comportamiento y en los hábitos de consumo del recurso hídrico en los habitantes; para ello, se analizaron las actitudes de las personas en el momento de aprovechar, captar o disponer del agua, incluidas las limitaciones que les impiden adoptar los cambios en los hábitos de consumo. Esta investigación, entonces, exploró el recurso hídrico (disponibilidad y calidad), uso de conductas y actitudes de todos los sectores de consumo ubicados en la zona urbana y rural del suroeste de Victoria. Las estrategias propuestas se dirigieron a trazar un modelo conceptual de los factores que impactan en el uso del agua de los usuarios, incluidos los hábitos de consumo y las limitaciones (económicas, infraestructura), con el cual se alcanzara un entendimiento para efectos del ahorro crucial y prioritario del agua en la región. Con esta investigación fue posible identificar que los siguientes factores influían en el consumo del recurso hídrico:

- Los usuarios y habitantes del suroeste de Victoria no tenían claro que la fuente (cuerpos de agua subterránea, cuerpos de agua superficial) de agua es la abastecedora para fines de desarrollo económico, social y cultural.

- $\quad$ No se percibió una noción en los usuarios sobre el nivel de escasez del agua.

- Existían confrontaciones sobre la responsabilidad del cuidado, conservación y monitoreo del agua entre las autoridades competentes del medio ambiente y el Gobierno.

- Se encontró una diferencia en los impulsores del ahorro de agua: de un lado se encontraban los agricultores querían ser "eficientes" en su consumo "para mantener su negocio viable y productivo"; mientras que los agricultores aficionados y los usuarios residenciales estaban "ahorrando agua" por razones más altruistas.

Estos hallazgos tienen implicaciones para el desarrollo de estrategias de gestión de la demanda en esta y otras áreas rurales y regionales. Sin embargo, el modelo 
conceptual debe ser objeto de pruebas para determinar si realmente refleja los factores que influyen en el comportamiento de ahorro de agua en dichas áreas [12].

De otro lado, la oficina regional del Instituto Internacional para el Manejo del Agua (IWMI, por sus iniciales en inglés) de Hyderabad (India) realizó una investigación sobre la productividad del agua y la huella hídrica, según los conceptos herramientas y políticas de la gestión del agua; el trabajo realizado dio lugar a proponer nuevos conceptos sobre el uso eficiente del recurso hídrico. Esta investigación partió de las preocupaciones y discusiones sobre la productividad del agua y la huella hídrica como conceptos técnicos aplicables al desarrollo económico de la ciudad, al igual que su validez tórica y valor práctico. Se estableció con este trabajo que la productividad del agua y la huella hídrica son directamente proporcionales y dependen de la finalidad del aprovechamiento y captación del agua, es decir, del contexto de uso y del interés. Una vez definida la importancia y el valor práctico de la productividad del agua y la huella hídrica, se estableció que se deben formular estrategias, políticas y una gestión del agua que involucre a todos los sectores de consumo [13].

\section{Materiales y métodos}

\subsection{Zona de estudio}

El departamento de Boyacá, uno de los 32 que componen el territorio colombiano, está localizado en la zona centro-nororiental del país; limita con los departamentos de Antioquia, Santander, Arauca, Casanare y Cundinamarca. El departamento se encuentra subdividido en provincias: el municipio de Pauna, lugar de estudio y desarrollo del proyecto, está localizado en la provincia occidental. Este recibe el nombre de "la puerta verde de Colombia" en tanto su cabecera municipal se encuentra localizada en la latitud $5^{\circ} 40^{\prime}$ norte y longitud $73^{\circ} 59^{\prime}$ oeste, en la parte alta de la zona esmeraldera de Colombia [3].

"La hidrología del municipio está conformada por las corrientes más importantes de la zona por su caudal, longitud y estructuras de paso son el río Minero, Ibacapí, quebrada Manotera, Paunera, Piache y Guayabal, las cuales han labrado cañones en forma de "v" suave. Se observan pequeños procesos de socavación y todas las corrientes finalmente confluyen al río Minero" [3]. 


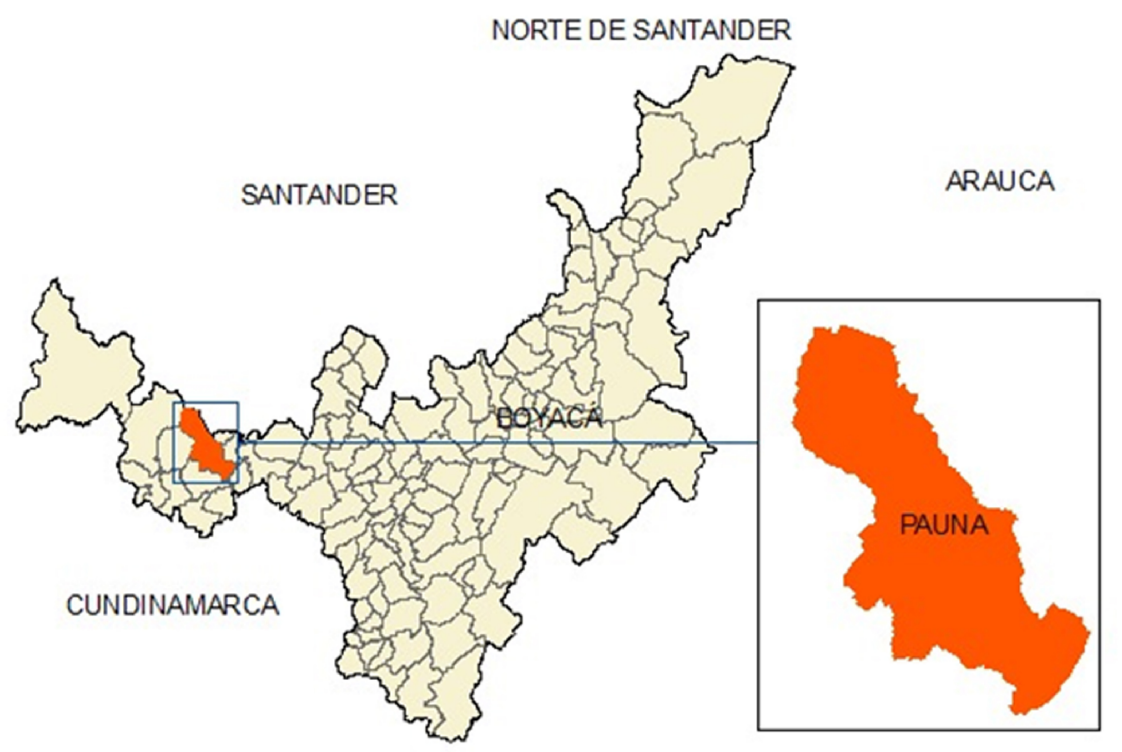

Figura 1. Ubicación del Municipio de Pauna

Fuente: Alcaldía Municipal del Municipio de Pauna [3]

"La red hidrológica del área es muy importante, dado que pertenece a la cuenca alta del río minero, el cual recorre al área de occidente, de sur a norte y recibe numerosos ríos y quebradas que tienen su origen en la parte alta del municipio de Pauna (zona de recarga hídrica), dentro de esta se tiene el río Ibacapí, que atraviesa el municipio de oriente a occidente, quebrada Guas, que separa a Pauna del municipio de Florián, quebradas Tortuga, Guayacán, Trementona, Agua Fría, Río de Oro, Río Bamba, Sabaleña, El Chamizo, El Loro, Amarilla, Agüita, La Honda, Hacomay, Paunera, Manotera, Tunera, Piache, Minera, la Bizca, Guayabalito, Toberia, Carmiazeña" [3].

\subsection{Parámetros físicos y químicos}

La identificación del estado de los cuerpos de agua como sistema ecológico requiere llevar a cabo una evaluación de la calidad del agua, la cual permite verificar la presencia de los parámetros que identifican la calidad del agua y el análisis respectivo. En la tabla 2 se muestran los parámetros físicos y químicos que se usaron para analizar la calidad del agua en el municipio estudiado. 
Tabla 2. Parámetros físicos, químicos y bacteriológicos

\begin{tabular}{|c|c|}
\hline Parámetros generales & Parámetros específicos \\
\hline \multirow{5}{*}{ Físico } & - Temperatura \\
\hline & - Color \\
\hline & - Turbiedad \\
\hline & - Sólidos totales disueltos (STD) \\
\hline & - Conductividad \\
\hline \multirow{5}{*}{ Químico } & $\cdot \mathrm{pH}$ \\
\hline & - Alcalinidad \\
\hline & - Acidez \\
\hline & - Dureza \\
\hline & - Oxígeno \\
\hline \multirow{2}{*}{ Orgánico } & - Demanda biológica de oxígeno (DBO) \\
\hline & - Demanda química de oxígeno (DQO) \\
\hline
\end{tabular}

Fuente: elaboración propia

\subsubsection{Temperatura}

La temperatura es un parámetro físico importante que incide en la solubilidad del oxígeno y las velocidades en el metabolismo, difusión y reacciones químicas y bioquímicas, al tiempo que afecta la mayoría de los procesos biológicos que tienen lugar en los ecosistemas acuáticos [14]. Una descarga no contaminante térmicamente debe tener una temperatura no superior a $25^{\circ} \mathrm{C}$ [15].

\subsubsection{Turbiedad}

Con este parámetro se mide la intensidad de la luz que pasa a través de la muestra por efecto de la materia suspendida y coloidal. La turbiedad puede ser causada por material finamente dividido en suspensión como arcilla, sílice, materia orgánica, sustancia mineral y, en general, por desechos industriales y domésticos [16].

\subsubsection{Sólidos totales disueltos (STD)}

A través de los STD se mide la cantidad de materia disuelta en el agua. Sus orígenes pueden ser múltiples, tanto en las aguas subterráneas como en las superficiales. Los STD comprenden las sales inorgánicas (calcio, magnesio, potasio y sodio, bicarbonatos, cloruros y sulfatos) y pequeñas cantidades de materia orgánica que están disueltas en el agua. 


\subsubsection{Conductividad}

Este parámetro, a través del cual se mide la capacidad del agua para conducir la electricidad, indica la materia ionizable presente en el agua. El agua pura prácticamente no conduce la electricidad; por lo tanto, la conductividad que sea posible medir será consecuencia de las impurezas presentes en el agua [16].

\subsection{5. $\mathrm{pH}$}

"Se define como el logaritmo de la inversa de la concentración de protones:

$$
\mathrm{pH}=\log 1 /[\mathrm{H}+]=-\log [\mathrm{H}+]
$$

El valor del pH compatible con la vida piscícola está comprendido entre 5 y 9; sin embargo, para la mayoría de las especies acuáticas la zona de $\mathrm{pH}$ favorable se sitúa entre 6,0 y 7,2; fuera de este rango no es posible la vida como consecuencia de la desnaturalización de las proteínas" [17].

\subsubsection{Alcalinidad}

Según el Instituto de Hidrología, Meteorología y Estudios Ambientales (Ideam), la alcalinidad es la capacidad de neutralizar ácidos, así como la suma de todas las bases titulables; asimismo, es la medida de una propiedad agregada del agua y puede interpretarse en términos de sustancias específicas. La alcalinidad es, ante todo, una función del contenido de carbonato, bicarbonato e hidróxido, las cuales son las bases más comunes de encontrar en las aguas superficiales [18].

\subsubsection{Acidez}

La acidez del agua es una medida de su capacidad para reaccionar con bases fuertes a determinado $\mathrm{pH}$; es decir, es la capacidad para donar protones. Se trata de una medición de las propiedades agregadas del agua y puede ser interpretada en términos de sustancias específicas; los ácidos minerales fuertes, los ácidos débiles (como carbónico y acético) y las sales hidrolizadas, tales como las ferrosas o los sulfatos de aluminio, pueden contribuir a la acidez del agua [18]. 


\subsubsection{Dureza}

La dureza se entiende en el agua como el contenido de metales alcalinotérreos, especialmente calcio y magnesio. Su importancia está concentrada en el hecho de que estos metales inhiben la acción de algunos agentes de tratamiento, impidiendo que el proceso de depuración de las aguas se lleve a cabo de manera apropiada [15].

\subsubsection{Oxígeno disuelto $(O D)$}

Esta medida permite determinar la actividad fisicoquímica y microbiológica de un sistema acuoso: su ausencia o bajo nivel se interpreta como un factor determinante del grado de contaminación del agua. Este parámetro previene o reduce el inicio de la putrefacción y la producción de cantidades objetables de sulfuros, mercaptanos y otros compuestos de mal olor. La determinación del OD sirve de base para el análisis de la DBO; así, tanto el primero como la segunda se consideran indicadores para medir el grado de contaminación de las aguas residuales domésticas e industriales [18].

De modo concreto, la DBO se expresa en mg / l; se define como la cantidad de oxígeno que los microorganismos, especialmente bacterias (aerobias-anaerobias facultativas: pseudomonas, escherichia, aerobacter, bacillius), hongos y plancton consumen durante la degradación de las sustancias orgánicas contenidas en la muestra [18]. A su turno, la demanda química de oxígeno (DQO) se define como la cantidad de oxígeno que requiere el agua para descomponer toda la materia orgánica que contiene. Es consumida por los cuerpos reductores presentes en el agua sin la intervención de los organismos vivos y efectúa la determinación del contenido total de materia orgánica oxidable, sea biodegradable o no [18].

\subsection{Metodologías desarrolladas}

La ejecución del proyecto se vio enmarcada en una investigación mixta, de caracteres cuantitativo (permitió la recolección de datos numéricos de las variables de estudio y su posterior análisis) y cualitativo (permitió la recolección de la información mediante encuestas y revisión de la documentación relevante al proyecto). El proceso adelantado comprendió, así, de una investigación documental de campo y de laboratorio mediante la realización de cuatro fases:

- Análisis de los instrumentos de planeación ambiental: se diagnosticaron y analizaron de manera integral los instrumentos de planeación ambiental relacionados con el consumo del agua. Pare ello, se adelantó un estudio 
cualitativo de aspectos relevantes de carácter ambiental, gestión del recurso hídrico y uso eficiente del agua, cuyo propósito fue recopilar información específica del municipio. Se solicitó ante la Secretaría de Planeación del municipio el Esquema de Ordenamiento Territorial y toda la documentación asociada (política ambiental, política de desarrollo sectorial turístico y sistema hídrico del municipio).

- Identificación y caracterización de los hábitos de consumo en los sectores comercial, residencial y turístico del municipio: se recolectó información sobre este aspecto mediante encuestas de forma participativa y tipo personal, y con preguntas abiertas, para obtener diferentes puntos de vista en cada uno de los sectores de consumo.

- Diagnóstico de la calidad del agua: se definió el plan de muestreo tipo puntual en cada una de las quebradas del municipio; se identificaron las propiedades del agua in situ; y se trasladaron las muestras al laboratorio, donde se realizó el análisis pertinente de la calidad del agua identificándose la caracterización fisicoquímica. Para efectos de asegurar la veracidad de los resultados, los equipos y materiales fueron calibrados en el laboratorio de agua de la Universidad Libre.

- La fase final consistió en formular estrategias de gestión para el uso eficiente del recurso hídrico en los sectores de consumo que fueron objeto de la investigación.

\section{Resultados}

\subsection{Resultados en campo}

Tabla 3. Resultados en campo, punto 1

\begin{tabular}{lcc}
\hline \multicolumn{2}{c}{ Nombre } & \multicolumn{2}{c}{ Coordenadas } \\
\hline \multirow{2}{*}{ Quebrada Manotera } & N & W \\
\cline { 2 - 3 } & $05^{\circ} 38,859^{\prime}$ & $073^{\circ} 58,663^{\prime}$ \\
\hline $\mathrm{pH}$ & Unidad & Resultado \\
\hline Turbidez & - & 6,59 \\
\hline Temperatura & $\mathrm{NTU}$ & 113 \\
\hline Conductividad & ${ }^{\circ} \mathrm{C}$ & 19,5 \\
\hline TDS & $\mathrm{NS} / \mathrm{cm}$ & 0,131 \\
\hline Oxígeno & $\mathrm{g} / \mathrm{l}$ & 0,068 \\
\hline
\end{tabular}

Fuente: elaboración propia 
Tabla 4. Resultados en campo, punto 2

\begin{tabular}{lcc}
\hline \multicolumn{1}{c}{ Nombre } & \multicolumn{2}{c}{ Coordenadas } \\
\hline \multirow{2}{*}{ Quebrada Murciera } & N & W \\
\cline { 2 - 3 } & $05^{\circ} 39,027$ & $073^{\circ} 58,808^{\prime}$ \\
\hline $\mathrm{PH}$ & Unidad & Resultado \\
\hline Turbidez & ---- & 6,53 \\
\hline Temperatura & $\mathrm{NTU}$ & 124 \\
\hline Conductividad & ${ }^{\circ} \mathrm{C}$ & 18,81 \\
\hline TDS & $\mathrm{MS} / \mathrm{cm}$ & 0,124 \\
\hline Oxígeno & $\mathrm{g} / \mathrm{l}$ & 0,064 \\
\hline
\end{tabular}

Fuente: elaboración propia

Tabla 5. Resultados en campo, punto 3

\begin{tabular}{lcc}
\hline \multicolumn{1}{c}{ Nombre } & \multicolumn{2}{c}{ Coordenadas } \\
\hline Cauce de separación & N & W \\
con la Quebrada Murciera & $05^{\circ} 39,102^{\prime}$ & $073^{\circ} 58,732^{\prime}$ \\
\cline { 2 - 3 } Parámetro & Unidad & Resultado $^{\prime}$ \\
\hline $\mathrm{PH}$ & --- & 6,13 \\
\hline Turbidez & $\mathrm{NTU}$ & 183 \\
\hline Temperatura & ${ }^{\circ} \mathrm{C}$ & 19,74 \\
\hline Conductividad & $\mu \mathrm{s} / \mathrm{cm}$ & 0,124 \\
\hline TDS & $\mathrm{g} / \mathrm{l}$ & 0,065 \\
\hline Oxígeno & $\mathrm{mg} / \mathrm{l}$ & 8,1 \\
\hline
\end{tabular}

Fuente: elaboración propia

Tabla 6. Resultados en campo, punto 4

\begin{tabular}{|c|c|c|}
\hline Nombre & & \\
\hline \multirow{2}{*}{ Quebrada Paunera } & $\mathbf{N}$ & w \\
\hline & $05^{\circ} 39,499^{\prime}$ & $073^{\circ} 58,547^{\prime}$ \\
\hline Parámetro & Unidad & Resultado \\
\hline $\mathrm{PH}$ & ------ & 5,87 \\
\hline Turbidez & NTU & 145 \\
\hline Temperatura & ${ }^{\circ} \mathrm{C}$ & 20,52 \\
\hline Conductividad & $\mu \mathrm{s} / \mathrm{cm}$ & 0,93 \\
\hline TDS & $\mathrm{g} / \mathrm{l}$ & 0,043 \\
\hline Oxígeno & $\mathrm{mg} / \mathrm{l}$ & 8,0 \\
\hline
\end{tabular}

Fuente: elaboración propia 


\subsection{Resultados de laboratorio}

Tabla 7. Resultados de laboratorio, punto 1

\begin{tabular}{|c|c|c|c|c|c|}
\hline \multicolumn{2}{|c|}{ Parámetro } & \multicolumn{2}{|c|}{ Unidad } & \multicolumn{2}{|c|}{ Resultado } \\
\hline \multicolumn{2}{|c|}{$\mathrm{PH}$} & \multicolumn{2}{|c|}{------ } & \multicolumn{2}{|c|}{7,23} \\
\hline \multicolumn{2}{|c|}{ Turbidez } & \multicolumn{2}{|c|}{ NTU } & \multicolumn{2}{|c|}{3} \\
\hline \multicolumn{2}{|c|}{ Temperatura } & \multicolumn{2}{|c|}{${ }^{\circ} \mathrm{C}$} & \multicolumn{2}{|c|}{17,23} \\
\hline \multicolumn{2}{|c|}{ Conductividad } & \multicolumn{2}{|c|}{$\mu \mathrm{s} / \mathrm{cm}$} & \multicolumn{2}{|c|}{0,131} \\
\hline \multicolumn{2}{|c|}{ TDS } & \multicolumn{2}{|c|}{$\mathrm{g} / \mathrm{l}$} & \multicolumn{2}{|c|}{0,066} \\
\hline \multicolumn{2}{|c|}{ Oxígeno } & \multicolumn{2}{|c|}{$\mathrm{mg} / \mathrm{l}$} & \multicolumn{2}{|c|}{7,0} \\
\hline DQO & DBO & Dureza & Hierro & Acidez & Alcalinidad \\
\hline 8 mg/l de 02 & $6,5 \mathrm{mg} / \mathrm{l}$ de $\mathrm{O} 2$ & $\begin{array}{c}85 \mathrm{mg} / \mathrm{l} \text { de } \\
\mathrm{CaCO} 3\end{array}$ & $0,28 \mathrm{mg} / \mathrm{l}$ & $\begin{array}{c}50 \mathrm{mg} / \mathrm{l} \mathrm{de} \\
\mathrm{CaCO} 3\end{array}$ & $\begin{array}{c}70 \mathrm{mg} / \mathrm{l} \mathrm{de} \\
\mathrm{CaCO} 3\end{array}$ \\
\hline
\end{tabular}

Fuente: elaboración propia

Tabla 8. Resultados de laboratorio, punto 2

\begin{tabular}{|c|c|c|c|c|c|}
\hline \multicolumn{2}{|c|}{ Parámetro } & \multicolumn{2}{|c|}{ Unidad } & \multicolumn{2}{|c|}{ Resultado } \\
\hline \multicolumn{2}{|c|}{$\mathrm{PH}$} & \multicolumn{2}{|c|}{------ } & \multicolumn{2}{|c|}{6,97} \\
\hline \multicolumn{2}{|c|}{ Turbidez } & \multicolumn{2}{|c|}{ NTU } & \multicolumn{2}{|c|}{1,5} \\
\hline \multicolumn{2}{|c|}{ Temperatura } & \multicolumn{2}{|c|}{${ }^{\circ} \mathrm{C}$} & \multicolumn{2}{|c|}{13,94} \\
\hline \multicolumn{2}{|c|}{ Conductividad } & \multicolumn{2}{|c|}{$\mu \mathrm{s} / \mathrm{cm}$} & \multicolumn{2}{|c|}{0,127} \\
\hline \multicolumn{2}{|c|}{ TDS } & \multicolumn{2}{|c|}{$\mathrm{g} / \mathrm{l}$} & \multicolumn{2}{|c|}{0,064} \\
\hline \multicolumn{2}{|c|}{ Oxígeno } & \multicolumn{2}{|c|}{$\mathrm{mg} / \mathrm{l}$} & \multicolumn{2}{|c|}{7,5} \\
\hline DQO & DBO & Dureza & Hierro & Acidez & Alcalinidad \\
\hline $16 \mathrm{mg} / \mathrm{l}$ de $\mathrm{O} 2$ & $8 \mathrm{mg} / \mathrm{l}$ de $\mathrm{O} 2$ & $\begin{array}{c}89 \mathrm{mg} / \mathrm{l} \mathrm{de} \\
\mathrm{CaCO} 3\end{array}$ & 0,3 mg/l & $\begin{array}{c}70 \mathrm{mg} / \mathrm{l} \mathrm{de} \\
\mathrm{CaCO} 3\end{array}$ & $\begin{array}{c}20 \mathrm{mg} / \mathrm{l} \mathrm{de} \\
\mathrm{CaCO} 3\end{array}$ \\
\hline
\end{tabular}

Fuente: elaboración propia

Tabla 9. Resultados de laboratorio, punto 3

\begin{tabular}{|c|c|c|c|c|c|}
\hline \multicolumn{2}{|c|}{ Parámetro } & \multicolumn{2}{|c|}{ Unidad } & \multicolumn{2}{|c|}{ Resultado } \\
\hline \multicolumn{2}{|c|}{$\mathrm{PH}$} & \multicolumn{2}{|c|}{----- } & \multicolumn{2}{|c|}{6,65} \\
\hline \multicolumn{2}{|c|}{ Turbidez } & \multicolumn{2}{|c|}{ NTU } & \multicolumn{2}{|c|}{2} \\
\hline \multicolumn{2}{|c|}{ Temperatura } & \multicolumn{2}{|c|}{${ }^{\circ} \mathrm{C}$} & \multicolumn{2}{|c|}{12,96} \\
\hline \multicolumn{2}{|c|}{ Conductividad } & \multicolumn{2}{|c|}{$\mu \mathrm{s} / \mathrm{cm}$} & \multicolumn{2}{|c|}{0,137} \\
\hline \multicolumn{2}{|c|}{ TDS } & \multicolumn{2}{|c|}{$\mathrm{g} / \mathrm{l}$} & \multicolumn{2}{|c|}{0,069} \\
\hline \multicolumn{2}{|c|}{ Oxígeno } & \multicolumn{2}{|c|}{$\mathrm{mg} / \mathrm{l}$} & \multicolumn{2}{|c|}{7,6} \\
\hline DQO & DBO & Dureza & Hierro & Acidez & Alcalinidad \\
\hline 16 mg/l de $\mathrm{O} 2$ & 10 mg/l de 02 & $\begin{array}{c}92 \mathrm{mg} / \mathrm{l} \mathrm{de} \\
\mathrm{CaCO} 3\end{array}$ & 0,39 mg/l & $\begin{array}{c}70 \mathrm{mg} / \mathrm{l} \text { de } \\
\mathrm{CaCO} 3\end{array}$ & $\begin{array}{c}40 \mathrm{mg} / \mathrm{l} \mathrm{de} \\
\mathrm{CaCO} 3\end{array}$ \\
\hline
\end{tabular}

Fuente: elaboración propia 
Tabla 10. Resultados de laboratorio, punto 4

\begin{tabular}{|c|c|c|c|c|c|}
\hline \multicolumn{2}{|c|}{ Parámetro } & \multicolumn{2}{|c|}{ Unidad } & \multicolumn{2}{|c|}{ Resultado } \\
\hline \multicolumn{2}{|c|}{$\mathrm{pH}$} & \multicolumn{2}{|c|}{----- } & \multicolumn{2}{|c|}{6,32} \\
\hline \multicolumn{2}{|c|}{ Turbidez } & \multicolumn{2}{|c|}{ NTU } & \multicolumn{2}{|c|}{1,7} \\
\hline \multicolumn{2}{|c|}{ Temperatura } & \multicolumn{2}{|c|}{${ }^{\circ} \mathrm{C}$} & \multicolumn{2}{|c|}{13,5} \\
\hline \multicolumn{2}{|c|}{ Conductividad } & \multicolumn{2}{|c|}{$\mu \mathrm{s} / \mathrm{cm}$} & \multicolumn{2}{|c|}{0,96} \\
\hline \multicolumn{2}{|c|}{ TDS } & \multicolumn{2}{|c|}{$g / l$} & \multicolumn{2}{|c|}{0,046} \\
\hline \multicolumn{2}{|c|}{ Oxígeno } & \multicolumn{2}{|c|}{$\mathrm{mg} / \mathrm{l}$} & \multicolumn{2}{|c|}{7,6} \\
\hline DQO & DBO & Dureza & Hierro & Acidez & Alcalinidad \\
\hline 32 mg/l de 02 & 30 mg/l de 02 & $\begin{array}{c}108 \mathrm{mg} / \mathrm{l} \text { de } \\
\mathrm{CaCO} 3\end{array}$ & $1,4 \mathrm{mg} / \mathrm{l}$ & $\begin{array}{c}80 \mathrm{mg} / \mathrm{l} \mathrm{de} \\
\mathrm{CaCO} 3\end{array}$ & $\begin{array}{c}30 \mathrm{mg} / \mathrm{l} \mathrm{de} \\
\mathrm{CaCO} 3\end{array}$ \\
\hline
\end{tabular}

Fuente: elaboración propia

\subsection{Análisis de la calidad del agua}

Tras varias visitas al municipio y al área de estudio, se elaboró el plan de muestreo compuesto de agua que se presenta a continuación. Para ello, se tomaron varias muestras en distintos momentos y se dispusieron en el mismo recipiente a intervalos regulares de tiempo (una hora) durante un periodo de cinco horas, programado para la composición de la muestra. El objetivo planteado con esto radicó en tomar muestras de agua de las diferentes quebradas del municipio para analizar la calidad del recurso.

\subsubsection{Plan de muestreo}

\subsubsection{Criterios para la selección del punto de muestreo}

- Acceso: el punto de muestreo debe estar en un lugar fácilmente accesible, con las vías de acceso vehicular y peatonal que sean necesarias, de tal manera que se facilite obtener las muestras y transportar la carga que implican los equipos y materiales de muestreo.

- Representatividad: el punto de recolección de las muestras debe ser lo más representativo posible de las características totales del cuerpo de agua, esto significa que el cuerpo de agua debe estar mezclado totalmente en el lugar de muestreo, relacionado específicamente con la turbulencia, velocidad y apariencia física, haciendo que la muestra sea lo más homogénea posible. 
- Importancia: el punto de muestreo debe tener relevancia con el abastecimiento para las piscinas de los balnearios y acueducto del municipio de Pauna.

\subsubsection{Procedimiento de toma de muestra}

- Cálculo del caudal del cuerpo hídrico a muestra.

- Llenado del recipiente de muestreo con una porción de agua del cuerpo hídrico muestreado.

- Registro de localización del punto de muestreo con GPS.

- Caracterización del sitio con fotografías.

- Identificación de la muestra.

- Toma de muestras: esta se realizó sumergiendo el envase de forma contraria al flujo, con lo cual se evitaría la inclusión de aire por flujo turbulento.

\subsection{Análisis de campo}

Después de tomadas las muestras en el cuerpo de agua, se midieron los siguientes parámetros in situ:

- Turbiedad

- Conductividad

- Temperatura

- Caudal

- Sólidos totales disueltos

\subsubsection{Análisis característico de las propiedades del agua}

Se recolectaron muestras en cuatro puntos estratégicos, según los lineamientos del plan de muestreo. En los cuatro puntos se midieron las propiedades del agua y, mediante un muestreo de tipo compuesto, se tomaron las muestras, las cuales se trasladaron a los laboratorios de la Universidad Libre para realizar el análisis pertinente. Los datos en campo se obtuvieron mediante el uso de los equipos que se presentan en la tabla 11, previamente calibrados con asesoría de ingenieros de la Universidad Libre para asegurar que se utilizaran de forma correcta y arrojaran datos confiables. 
Se prepararon todos los equipos y materiales una semana antes y se realizó la gestión del traslado externo de equipos y materiales. Se definieron los puntos de muestreo de acuerdo con la facilidad de acceso a la zona y el interés de la recolección de la muestra; asimismo, fue definida la hora inicial en cada uno de los puntos. En la preparación de los envases se revisaron detenidamente cada uno de ellos para verificar que no presentaban fracturas y que el sellamiento del envase fuera el correcto; para los demás equipos como cámara digital, cinta métrica, GPS y formatos de campo que no necesitan calibración se verificó su correcto funcionamiento (tabla 11).

Tabla 11. Equipos.

\begin{tabular}{|c|c|c|}
\hline Equipo & Parámetro & Fotografía \\
\hline $\begin{array}{l}\text { Multiparámetro Horiba } \\
\text { u-50 }\end{array}$ & $\begin{array}{l}\text { pH, conductividad, temperatura, } \\
\text { turbidez, STD, ORP }\end{array}$ & \\
\hline Oxímetro YSI & Oxígeno disuelto & \\
\hline GPS Garmin Etrex 20X & Localización N W & \\
\hline
\end{tabular}

Envases para la toma de las muestras
Cantidad en $\mathrm{ml}$ del recurso

(capacidad de cada recipiente: un litro)

Fuente: elaboración propia 
Los métodos utilizados para determinar los parámetros del agua se presentan en la tabla 12.

Tabla 12. Métodos de análisis

\begin{tabular}{ll}
\hline \multicolumn{1}{c}{ Parámetro } & \multicolumn{1}{c}{ Método } \\
\hline Temperatura & ME-2550 B, método de laboratorio y campo \\
\hline Color & ME-2120 B, método de comparación visual \\
\hline Turbiedad & ME-2130 B, método nefelómetro \\
\hline Sólidos totales disueltos & ME-2540 C, sólidos totales disueltos secados a $180^{\circ} \mathrm{C}$ \\
\hline Conductividad & ME-2510 B, método de laboratorio \\
\hline Alcalinidad & ME-2320 B, método de titulación \\
\hline Acidez & ME-2310 B, método de titulación \\
\hline Dureza & ME-2340 B, dureza por cálculo \\
\hline Hierro & ME-3500Fe B, método de fenantrolina \\
\hline DQO & ME-5220 C, reflujo cerrado titrimétrico \\
\hline DBO & ME-5210 B, prueba DBO 5 días \\
\hline
\end{tabular}

Fuente: elaboración propia

De conformidad con la preservación y conservación de muestras de aguas, se estableció para cada uno de los parámetros, la metodología de preservación y su indicador respecto al tiempo; en la tabla 13 se presenta esta información en detalle.

Tabla 13. Preservación y almacenamiento de muestras de aguas

\begin{tabular}{ccc}
\hline Parámetro & Preservación & $\begin{array}{c}\text { Máximo almacenamiento } \\
\text { recomendado/regulatorio }\end{array}$ \\
\hline Temperatura & Analizar inmediatamente & $0,25 \mathrm{~h} / 0,25 \mathrm{~h}$ \\
\hline Color & Refrigeración & $48 \mathrm{~h} / 48 \mathrm{~h}$ \\
\hline Turbiedad & $\begin{array}{c}\text { Analizar el mismo día, guardar en oscu- } \\
\text { ridad hasta 24 horas; refrigerar }\end{array}$ & $24 \mathrm{~h} / 48 \mathrm{~h}$ \\
\hline Solidos totales disueltos & Refrigeración & $7 \mathrm{~d} / 2-7 \mathrm{~d}$ \\
\hline Conductividad & Refrigeración & $28 \mathrm{~d} / 28 \mathrm{~d}$ \\
\hline $\mathrm{pH}$ & Analizar inmediatamente & $0,25 \mathrm{~h} / 0,25 \mathrm{~h}$ \\
\hline Alcalinidad & Refrigeración & $24 \mathrm{~h} / 14 \mathrm{~h}$ \\
\hline Dureza & Adicionar HNO3 a pH $<2$ & $6 \mathrm{meses} / 6 \mathrm{meses}$ \\
\hline $\mathrm{DQO}$ & Analizar tan pronto sea posible, o adi- \\
cionar H2SO4 a pH <2.0, refrigerar & $7 \mathrm{~d} / 28 \mathrm{~d}$ \\
\hline $\mathrm{DBO}$ & Refrigeración & $6 \mathrm{~h} / 48 \mathrm{~h}$ \\
\hline
\end{tabular}

Fuente: elaboración propia 
En cuanto a la variación del campo al laboratorio, se deben tener en cuenta las siguientes observaciones:

- Para pH, la variación es debida al método de análisis.

- Para la turbiedad, la variación se debe a que en laboratorio se decantan las masas.

- El oxígeno varía en función de la altura y temperatura del lugar.

\subsection{Formulación de estrategias de gestión integrada y sostenible del recurso hídrico}

\subsubsection{Estrategia 1: control y vigilancia del consumo del agua}

- Objetivo: disminuir el consumo del recurso hídrico.

- Impacto ambiental: agotamiento del recurso hídrico.

- Actividades que causan el impacto:

- Uso de grifería obsoleta

- Uso insostenible del recurso hídrico

- Creciente demanda del agua para desarrollo y turismo

- Mantenimiento general de balnearios

- Cambio del agua de las piscinas de los balnearios

\subsubsection{Medidas de gestión}

Plano tecnológico: se requiere cambiar las tecnologías en uso, actualmente obsoletas, por tecnologías ahorradoras en los escenarios de alto consumo de agua. Para ello, es importante capacitar a los usuarios consumidores, así como a los organismos encargados de la gestión integral y sostenible del recurso hídrico, en materia de mecanismos para la conservación y ahorro del agua, y resaltar en ese sentido la importancia de su conservación y los métodos con los que se puede lograr. Con lo anterior, se consideró de la siguiente manera la tecnología que ayudaría a la conservación y buen uso del recurso hídrico:

- Accesorio de llave de lavaplatos ahorradora de agua (economiza 60 \%)

- Grifo pulsador para ducha

- Economizador para grifo de cocina (modelos hembra y macho). Regulador de caudal 
- Ducha monocontrol bahía

- Inspección y reparación de fugas: cada propietario deberá realizar una inspección detallada de las tuberías y verificar que no se presenten fugas; si estas ocurren, corresponde realizar las reparaciones a las que hubiere lugar de inmediato.

- Verificar el funcionamiento de los tanques de reserva; incentivar su instalación en los lugares que carezcan de ellos; y garantizar que su capacidad logre abastecer a los usuarios en periodos en los que haya corte del servicio por mantenimiento o por fallas de la planta de potabilización.

Plano social: educación ambiental (dos capacitaciones al mes para funcionarios y administrativos) respecto de los balnearios, que se realizará en el auditorio del municipio. Los temas que se llevarían a cabo en las capacitaciones se presentan a continuación:

- Primera capacitación

- Uso eficiente del recurso hídrico

- Sostenibilidad

- Tecnologías y equipos de ahorro

- Segunda capacitación

- Normatividad ambiental legal vigente (consumo del agua)

- Aspectos e impactos ambientales referidos al recurso hídrico

- Buenas prácticas para el ahorro del recurso hídrico

\section{Indicador:}

$\mathrm{m}^{3}$ de agua consumidos por mes
Habitante del municipio
$\mathrm{m}^{3}$ de agua consumidos por mes
Número de usuarios por mes




\subsubsection{Estrategia 2: formulación de estrategias de gestión ambiental, consumo del agua, control y vigilancia de la calidad del agua}

- Objetivo: disminuir la contaminación del agua.

- Impacto ambiental: Contaminación del agua.

- Actividades que causan el impacto:

- Generación de aguas residuales

- Usos de fertilizantes y pesticidas en las áreas agrícolas

- Desperdicios orgánicos e inorgánicos arrojados en las alcantarillas que desembocan en las quebradas del municipio.

- Desperdicios orgánicos e inorgánicos arrojados directamente en las quebradas del municipio.

\subsubsection{Medidas de gestión}

\section{Plano tecnológico:}

- Verificación del funcionamiento de la planta de tratamiento de aguas residuales (PTAR), ubicada en la vereda Ibama del municipio y que desde la fecha de construcción en el año del 2007 no ha tenido un correcto funcionamiento, en la actualidad se evidencia en el abandono, es necesario acorde a la generación de agua residual de los sectores de consumo colocarla en funcionamiento.

- Instalar trampas de grasas en cocinas y zonas de lavado.

- Analizar de forma mensual el agua de cada una de las quebradas en laboratorio, según los indicadores de la normatividad ambiental legal y los índices de calidad del recurso hídrico.

- Establecer un plan de manejo de residuos y realizar control y seguimiento de cada uno de los sectores de consumo.

- La administración municipal debe ejercer la función de control ambiental; y desde la oficina de Corpoboyacá (regional de occidente) se deben establecer geográficamente los puntos de vertimientos, así como realizar análisis y evaluación de la carga contaminante. Además, la alcaldía municipal y las autoridades ambientales competentes deben proceder a imponer sanciones ambientales por vertimientos y generación de residuos contaminantes del recurso hídrico. 


\section{Plano social:}

- Capacitaciones a los sectores de consumo, en convenio con voluntarios y comunidad estudiantil, sobre manejo de aceites usados, disposición final de los residuos sólidos y separación adecuada de los residuos.

- Implementar un programa de posconsumo como estrategia dirigida a promover la gestión ambientalmente adecuada de los residuos posconsumo, con el fin de que estos se sometan a sistemas de gestión diferencial y se evite que la disposición final se realice de manera incorrecta o en fuentes hídricas del municipio, como es el caso actual.

Tabla 14. Indicadores de calidad ambiental que se deben verificar y evaluar periódicamente

\begin{tabular}{|c|c|}
\hline Parámetros generales & Parámetros específicos \\
\hline \multirow{5}{*}{ Físico } & Temperatura \\
\hline & Color \\
\hline & Turbiedad \\
\hline & Sólidos totales disueltos (STD) \\
\hline & Conductividad \\
\hline \multirow{6}{*}{ Químico } & $\mathrm{pH}$ \\
\hline & Alcalinidad \\
\hline & Acidez \\
\hline & Hierro \\
\hline & Dureza \\
\hline & Oxígeno \\
\hline \multirow{2}{*}{ Orgánico } & DBO \\
\hline & DQO \\
\hline
\end{tabular}

Fuente: elaboración propia

\section{Discusión}

La investigación realizada pretende incentivar las políticas públicas de planeación, que no se reportan en el territorio estudiado pese al hecho de que son necesarias. En este sentido, los sectores residencial, comercial y turístico no miden el consumo de acuerdo con las estrategias establecidas, sino por la demanda ocasionada en el curso natural de sus operaciones, hecho que desemboca en el uso ineficiente del agua. Partiendo del análisis de las investigaciones realizadas a nivel nacional respecto de 
la formulación de estrategias de gestión integrada del recurso hídrico, la presente investigación enfocó sus esfuerzos a proponer estrategias dirigidas a la vigilancia, control y monitoreo de la calidad y consumo de agua para cada uno de los sectores de consumo involucrando la administración municipal, algo que no se observó en las investigaciones que compusieron el estado del arte. Ahora bien, el presente proyecto resulta insuficiente para integrar una municipalidad de mayor extensión; sin embargo, los aportes técnicos expuestos deberían ser tenidos en cuenta al emprender cualquier proyecto de esta índole.

Para garantizar la realización de las capacitaciones establecidas en las estrategias de gestión ambiental fue preciso recurrir a las dependencias de responsabilidad social y ambiental de las instituciones o empresas inmersas en los temas ambientales; estas suelen incluir en su agenda programas de socialización, a través de los cuales se pretende informar a los consumidores y la población en general sobre la importancia de los recursos hídricos y las estrategias ambientales para su conservación. Así entonces, para hacer sostenible el plan de capacitación y las estrategias formuladas se acudió a las diferentes instituciones en pro de que la socialización estas últimas no acarreara costo alguno para los usuarios o la administración municipal. Las capacitaciones contaron con la participación y presencia de funcionarios de la oficina de Corpoboyacá (regional occidente) del municipio de Pauna, de la administración departamental, del concejo y de la administración municipal, al tiempo que concurrieron rectores y representantes estudiantiles de las instituciones educativas y de la Universidad Libre, y líderes de la región; estos compartieron, sin interés diferente al cultural, las herramientas metodológicas formuladas.

\section{Conclusiones}

La calidad del agua en el municipio de Pauna se encuentra afectada por los usos inadecuados del recurso hídrico. A ello se suman factores como la falta de un plan de manejo de residuos: el desconocimiento de la población, que se deshace de los residuos en los alrededores de las quebradas, quedó demostrado a través de las encuestas.

El análisis sobre la calidad del agua demuestra que las quebradas del municipio de Pauna no presentan índices de contaminación. Excepción a lo anterior es la quebrada "La Paunera", que está ubicada en las proximidades de las industrias de porcicultura; esto causa un impacto negativo en la calidad del recurso, situación que debe ser controlada por la oficina regional de Corpoboyacá que se encuentra ubicada en la municipalidad. 
La captación del agua para la planta de potabilización del municipio estudiado procede de la Quebrada Manotera. Esta última ostenta condiciones óptimas para el consumo del agua; empero, una vez esta ha recorrido una distancia de aproximadamente un kilómetro abajo de la planta de potabilización, no presenta las mismas condiciones debido al material de arrastre, la generación de residuos y el uso de fertilizantes y pesticidas.

En términos generales, la población no ha recibido capacitación sobre estrategias de uso eficiente del recurso hídrico, por lo que el consumo del mismo por parte de los diferentes sectores resulta elevado -y cabe resaltar el sector de mayor consumo es el turístico-. La realización de las encuestas, dejó constancia de la inobservancia de los conocimientos de sostenibilidad en temas de ahorro y uso eficiente del recurso hídrico, en tanto no es un tema de interés o relevancia hasta el momento. Una vez realizado el diagnóstico y puesta en contexto la gestión integrada del recurso, la población en general se mostró interesada en recibir asesoría, capacitación y aplicación de estrategias en temas afines al uso eficiente del recurso en cuestión.

De los resultados expuestos se destaca que el agua se encuentra en condiciones óptimas para su aprovechamiento por parte de la industria, así como para el consumo humano. Con esto, las estrategias elaboradas en el marco de este proyecto requieren una trazabilidad permanente porque, si bien desde el enfoque socioambiental evitan la escasez y el agotamiento del recurso hídrico en virtud de la capacidad de agua con la que cuenta el municipio, no existe garantía alguna de que se haga uso sostenible de la misma.

La carencia de herramientas para el uso adecuado del recurso hídrico en el territorio analizado queda en evidencia con las diferentes pruebas técnicas que se han aportado mediante el presente trabajo. A su vez, y como se dijo, la construcción turística de los denominados balnearios ha causado el aumento del consumo del recurso hídrico; no obstante, la formulación de las políticas administrativas que se encuentran en cabeza de la administración municipal ha resultado insuficiente ante el crecimiento en la demanda del agua. Así entonces, las circunstancias expuestas han derivado en la formulación de estrategias de gestión integrada y sostenible del recurso hídrico en el municipio de Pauna.

\section{Referencias}

[1] J. Arboleda, Manual para la evaluación de impacto ambiental de proyectos, obras o actividades. Medellín, pp 13-29, 2008 [En línea]. Disponible en: https://www.kpesic.com/sites/default/ files/Manual_EIA_Jorge\%20Arboleda.pdf 
[2] C. Zamudio Rodríguez, "Gobernabilidad sobre el recurso hídrico en Colombia: entre avances y retos”, Gestión y ambiente, vol. XV, n. ${ }^{0}$ 3, pp. 4-5, 2012. [En línea]. Disponible en: https:// revistas.unal.edu.co/index.php/gestion/article/view/36284/42930

[3] Alcaldía Municipal del Municipio de Pauna, Esquema de ordenamiento territorial, EOT. Pauna, Boyacá, 2014.

[4] M. C. Bustamante, K. L. Cárdenas y J. L. Corredor, Formulacion del Programa de Ahorro y Uso Eficiente de Agua para la Empresa de Servicios Publicos del Municipio Gachetá - Cundinamarca. Bogotá: Universidad Militar Nueva Granada, pp. 1-22, 2011. [En línea]. Disponible en: http:// www.umng.edu.co/documents/10162/1299317/ART_21.pdf

[5] A. M. B. Panche, K. P. Zabaleta Rodríguez y Á. Chávez Porras, Programa de ahorro y uso eficiente del agua para la empresa Empucol del Municipio El Colegio, Cundinamarca. Bogotá: Universidad Militar Nueva Granada, pp. 1-20, 2012 [En línea]. Disponible en: http://www. umng.edu.co/documents/10162/745281/V3N2_3.pdf

[6] J. F. Franco Barrera, Estrategia de gestión ambiental a partir de la formulación de un esquema de pago de servicios ambientales (PSA) para la regulación del recurso hídrico en la quebrada "La Colorada" del municipio de Arcabuco (Boyacá). Bogotá: Pontificia Universidad Javeriana, pp. 72-89, 2009 [En línea]. Disponible en: https://repository.javeriana.edu.co/bitstream/handle/10554/728/eam47.pdf?sequence=1\&isAllowed=y

[7] Universidad Tecnológica de Pereira, Estrategias de Uso Eficiente y ahorro de agua en Centros Educativos. Pereira: Universidad Tecnológica de Pereira, pp. 23-83, 2012 [En línea]. Disponible en: http://repositorio.utp.edu.co/dspace/bitstream/handle/11059/2769/333912T866.pdf? sequence $=1 \&$ isAllowed=y

[8] J. Carvajal Escobar, "La gestión integrada de los recursos hídricos como estrategia de adaptación al cambio climatico", Ingeniería y competitividad, vol. 9, n. ․ 1, pp. 19-29, 2007 [En línea]. Disponible en: https://www.portalces.org/sites/default/files/migrated/docs/La_gestion_integrada_de_los_recursos_hidricos_como_estrategia_de_adaptacion_al_C.C_\%28Martha_ Garcia_et_al.\%29.pdf

[9] I. Restrepo Tarquino, "Tendencias Mundiales en la Gestión de Recursos Hídricos: Desafíos para la Ingeniería del Agua”, Ingeniería y competitividad, vol. 6, n. 1, pp. 63-71, 2014. doi: https://doi.org/10.25100/iyc.v6i1.2289

[10] A. L. Hernández Matiz, Ahorro y uso eficiente del recurso agua en una empresa del sector de aceites y grasas vegetales. Bogotá: Universidad de la Salle, pp. 34-104, 2007 [En línea]. Disponible en: http://repository.lasalle.edu.co/bitstream/handle/10185/14043/T41.07\%20H43a.pdf 
[11] Australian Demographic Statistics, "Australian Bureaun of Statistic", 2017. [En línea]. Disponible en: http://www.abs.gov.au/ausstats/abs@.nsf/mf/3101.0/ [Consultado el 26 de Nov. de 2017].

[12] M. L. M. Graymore y A. M. Wallis, "Water savings or water efficiency? Water-use attitudes and behaviour in rural and regional areas", International Journal of Sustainable Development \& World Ecology, vol. 17, n. ${ }^{\circ}$ 1, pp. 84-93, 2010. doi: https://doi.org/10.1080/13504500903497249

[13] U. A. Amarasinghe y V. Smalkhtin, "Water productivity and water footprint: misguided concepts or useful tools in water management and policy", Water International, vol. 39, n. ${ }^{\circ}$ 7, pp. 1000-1017, 2014. doi: https://doi.org/10.1080/02508060.2015.986631

[14] A. Cirelli, "El agua: Un recurso esencial", Quimica viva, vol. 11, n. 3 3, pp. 147-170, 2012 [En línea]. Disponible en: http://www.redalyc.org/pdf/863/86325090002.pdf

[15] J. Rojas, Tratamiento de aguas residuales: teoría y principios de diseño. Bogotá: Escuela Colombiana de Ingeniería, 2000, pp. 35-56.

[16] A. Playeras, "Parámetros de Calidad de las Aguas de Riego", Bonsai Menorca, 2011 [En línea]. Disponible en: http://www.bonsaimenorca.com/articulos/articulos-tecnicos/parametros -de-calidad-de-las-aguas-de-riego/

[17] E. Pérez López, "Control de calidad en aguas para consumo humano en la región occidental de Costa Rica". Tecnología en Marcha, vol. 29, n. 3, pp. 3-14, 2016. doi: https://doi. org/10.18845/tm.v29i3.2884

[18] C. Gómez Gallego, Métodos Analíticos para la Evaluación de la Calidad Fisicoquímica del Agua. Manizales: Universidad Nacional de Colombia, 2010, pp. 26-74. http://bdigital.unal. edu.co/49658/7/9789588280394.pdf. 